\title{
Philosophiques
}

\section{Duncan Pritchard. Scepticism : A Very Short Introduction, New York, Oxford University Press, 2019, 116 pages}

\section{Thierry Laisney}

Volume 47, numéro 1, printemps 2020

URI : https://id.erudit.org/iderudit/1070259ar

DOI : https://doi.org/10.7202/1070259ar

Aller au sommaire du numéro

Éditeur(s)

Société de philosophie du Québec

ISSN

0316-2923 (imprimé)

1492-1391 (numérique)

Découvrir la revue

Citer ce compte rendu

Laisney, T. (2020). Compte rendu de [Duncan Pritchard. Scepticism : A Very

Short Introduction, New York, Oxford University Press, 2019, 116 pages].

Philosophiques, 47(1), 235-238. https://doi.org/10.7202/1070259ar d'utilisation que vous pouvez consulter en ligne.

https://apropos.erudit.org/fr/usagers/politique-dutilisation/ 
rique serait advenu, entre autres, de l'intérieur du schème traditionnel, ce dont l'ensemble de l'ouvrage témoigne, apparaît en porte-à-faux avec l'ontologie chaotique castoriadienne. Or, précise l'auteur en introduction, celleci, faite de ruptures radicales comme créations de formes non dérivables du déjà-là sous-tend le régime historique. Les "voies contournées» (p. 90) par lesquelles les schèmes conceptuels adviennent font certes signe vers une conception elle-même discontinue de l'historiographie, mais semblent bien loin de l'ontologie chaotique castoriadienne, qui ne voit que «solution de continuité» (p. II) entre les sociétés et leurs systèmes d'idées. Blumenberg, également mentionné brièvement en introduction, serait sans doute plus à propos pour rendre compte de l'avènement inhomogène du régime historique fondé sur la discontinuité.

GENEVIÈVE GENDREAU

Université d'Ottawa

\section{Duncan Pritchard. Scepticism: A Very Short Introduction, New York, Oxford University Press, 2019, 116 pages.}

Avec ce livre, Scepticism: A Very Short Introduction, le professeur Duncan Pritchard, l'une des figures majeures de l'épistémologie contemporaine, s'est prêté à un exercice plutôt difficile: traiter en une centaine de pages un sujet vieux comme la philosophie. Pritchard met l'accent sur une forme particulière de scepticisme, le scepticisme radical, qui a pour but de nous faire révoquer en doute la moindre de nos croyances. Il examine les arguments qui jouent en faveur de ce scepticisme radical et ceux qui ont pu être avancés pour le rejeter.

Pritchard ne cite pas Malebranche, mais il pourrait faire sienne la distinction que ce dernier opère entre deux sortes de doute:

[I]l y a bien de la différence entre douter et douter. On doute par emportement et par brutalité, par aveuglement et par malice; et enfin par fantaisie et parce que l'on veut douter. Mais on doute aussi par prudence et par défiance, par sagesse et par pénétration d'esprit. Les académiciens et les athées doutent de la première sorte, les vrais philosophes doutent de la seconde: le premier doute est un doute de ténèbres, qui ne conduit point à la lumière, mais qui en éloigne toujours; le second doute naît de la lumière, et il aide en quelque façon à la produire à son tour ${ }^{1}$.

Ce «doute de lumière» s'accorde avec ce que Pritchard appelle le "scepticisme modéré», qu'il met au rang des vertus intellectuelles. Pour reprendre les exemples de l'auteur, il est raisonnable de ne pas ajouter foi

1. Nicolas Malebranche, De la recherche de la vérité, Paris, Charpentier, I842, livre I, chapitre XX, p. 93. 
aux prédictions des horoscopes, ou bien de manifester une certaine défiance à l'endroit des vendeurs de voitures d'occasion; il sera encore judicieux de ne pas prendre pour argent comptant l'affirmation selon laquelle la reine d'Angleterre vient d'être arrêtée pour vol à l'étalage. Le scepticisme modéré est un antidote à la crédulité. Or Pritchard souligne qu'une attitude parfaitement crédule reviendrait à ne détenir aucune connaissance. Connaître, en effet, ce n'est pas seulement avoir une croyance vraie: c'est aussi avoir de bonnes raisons de croire, des raisons épistémiques et non prudentielles (un revolver sur la tempe, il me sera utile de croire à la religion de paix dont on veut me persuader, mais je ne croirai pas alors pour de bonnes raisons; à vrai dire, je feindrai seulement de croire). Une personne qui serait totalement crédule ne formerait des croyances vraies que par hasard (il est difficile d'imaginer que quelqu'un n'entende que des propositions fausses) et ne saurait donc rien à proprement parler.

Cette absence totale de connaissance, la crédulité la partage avec un scepticisme généralisé. Pritchard relève que, contrairement au doute localisé qui se fonde sur ce que nous savons par ailleurs, ce type de doute massif qui, d'autre part, conduirait à une existence dépourvue de sens - ne se fonde plus sur rien. Si plus rien n'est tenu pour vrai, on aboutit à un relativisme à l'égard de la vérité. L'attitude relativiste, note Pritchard, peut s'expliquer de plusieurs façons: certaines questions sont affaire d'opinion personnelle (de gustibus...); le relativisme peut être interprété (faussement) comme une façon de respecter les vues des autres; nos jugements ne sont ni infaillibles ni certains. Mais l'auteur observe que la certitude n'est pas une condition de la connaissance. Je peux dire que je sais que ma voiture est garée devant chez moi car j'ai de très bonnes raisons de le croire. Mais, après tout, je n'en suis pas absolument sûr: elle a peut-être été volée. Si tel est le cas, je saurai après vérification que ma croyance était fausse, mais elle n'en était pas moins justifiée. Le caractère faillible et incertain de nos croyances ne peut donc autoriser un scepticisme à tout-va.

L'auteur montre que le scepticisme radical s'attaque moins à la vérité de nos croyances qu'à leur bien-fondé. Les hypothèses sceptiques radicales trouvent leur source dans l'œuvre de Descartes (qui n'y recourait pas à des fins sceptiques, mais au contraire pour établir ce qui est indubitable) et présentent, selon Pritchard, deux caractéristiques principales: elles envisagent des expériences qui ne se distinguent en rien des expériences que nous vivons tous les jours; elles rendent fausses la plupart de nos croyances, celles en particulier qui sont relatives au monde extérieur. Dans la première de ses Méditations métaphysiques, Descartes expose deux hypothèses de ce genre: notre vie n'est peut-être qu'un rêve; peut-être un "mauvais génie » cherchet-il (et parvient-il) à nous tromper (même au sujet des vérités mathématiques les plus élémentaires). L'expérience de pensée selon laquelle nous serions des cerveaux dans une cuve reliés à un ordinateur est une variante moderne de ces hypothèses. 
Le problème pour qui veut s'opposer au scepticisme radical est que ces hypothèses ne sont pas réfutables, puisque les expériences qui leur sont associées ne diffèrent aucunement de celles de notre vie quotidienne. Pire, le sceptique radical va pouvoir user d'une arme redoutable pour renforcer sa thèse: la clôture épistémique (si vous savez que $p$ et que vous savez que $p \rightarrow q$, alors vous savez aussi que $q$ ). Un exemple emprunté à Pritchard permettra de le comprendre. Je pense savoir que je porte une chemise. Mais si c'est vrai, il est vrai que je ne suis pas un cerveau dans une cuve (un cerveau ne porte pas de chemise). Or il m'est impossible d'écarter cette dernière hypothèse! Cette contradiction permet au sceptique de prétendre qu'en réalité nous ne savons rien: nos raisons de croire ne sont de bonnes raisons qu'en apparence. Pour résumer la situation (et le cœur du livre), les trois affirmations suivantes ne peuvent être vraies ensemble: nous sommes incapables de réfuter les hypothèses sceptiques radicales (a); nous devons tenir compte de la clôture épistémique (b); nous assumons, dans notre vie de tous les jours, la connaissance de quantité de choses (c). À ce stade, une distinction essentielle va être faite par Pritchard. Ou bien nous considérons le scepticisme radical comme étant une position, et nous déclarerons alors que la proposition (c) est fausse. Ou bien nous le considérons comme énonçant un paradoxe, et il nous sera loisible dans ce cas d'infléchir notre conception du savoir dans le sens de la résolution de cette incohérence.

Que répondre au défi sceptique? Le recours au sens commun peut constituer une première défense. Pour G. E. Moore, des énoncés du sens commun ("Voici une main ", par exemple) sont plus certains que n'importe quel argument philosophique visant à les démentir. Selon lui, nous devons souscrire à la proposition (c) et, au moyen du principe de clôture, conclure que la réfutation des hypothèses sceptiques radicales est possible. Le problème est que Moore n'est pas en mesure d'expliquer pourquoi (a) est fausse: le sens commun nous dicte que cette proposition doit être fausse, et on ne peut pas en dire davantage.

Une autre approche consisterait à faire du verbe "savoir» un terme sensible au contexte: selon les cas, "savoir" correspondrait à une exigence épistémique plus ou moins forte. De cette façon, les trois propositions mentionnées plus haut ne seraient plus vraiment en conflit puisqu'elles ne parleraient plus tout à fait de la même chose. Mais le contextualisme peut sembler (Pritchard est moins sévère que cela) une réponse un peu trop facile au paradoxe sceptique. Le rapprochement avec les «indexicaux» (qui, eux, sont manifestement «sensibles au contexte», pensons aux pronoms personnels ou à des adverbes comme «ici» ou "maintenant») a quelque chose d'artificiel. Quant aux adjectifs comme "grand» que cite également l'auteur (certes, "grand" ne s'entend pas de la même manière pour un joueur de basket et pour un individu lambda), ne relèvent-ils pas davantage du "vague» que du contextualisme? L'examen du verbe "savoir» peut nous inviter (l'auteur ne prend pas ce chemin) à un petit détour par la lexicologie. 
Si ce mot a véritablement un sens strict et un sens plus lâche, pourquoi ne pas parler de polysémie plutôt que de «sensibilité au contexte»? Enfin, et sans parler de l'évolution en intensité du sens des mots, la plupart d'entre eux voient leur signification légèrement modifiée par le contexte de leur utilisation: "savoir » n'est pas un cas à part. Le contextualisme ne semble donc pas un argument très probant pour contrer le défi sceptique.

Pour tenter de le faire, Pritchard va faire appel ensuite à Wittgenstein et à son ouvrage De la certitude. Si Moore affirmait (sans preuve) la fausseté de la proposition (a), Wittgenstein dénie, quant à lui, la proposition (b), c'est-à-dire la clôture épistémique. Pour Wittgenstein, l'erreur des sceptiques radicaux (comme de ceux qui, traditionnellement, les combattent) consiste à produire des évaluations rationnelles globales de nos croyances; nous n'évaluons jamais toutes nos croyances en bloc, mais seulement une partie d'entre elles que nous rapportons à des certitudes du sens commun qui, elles, sont a-rationnelles. La pratique même de l'évaluation rationnelle des croyances présuppose de telles certitudes, que Wittgenstein compare aux gonds d'une porte (il faut qu'ils soient fixes pour qu'elle tourne) et qui, ne relevant pas du savoir, échappent au travail de sape du sceptique radical. Comme pour les deux premières réponses, il serait difficile de voir dans la position de Wittgenstein la résolution définitive du paradoxe sceptique. Il n'y a pas de solution bien nette. Sans doute (si j'ose dire) faut-il se contenter — Pritchard ne le suggère pas en ces termes — de ce qu'on pourrait appeler un « internalisme épistémique » : nous savons beaucoup de choses dans notre vie de tous les jours tout en ignorant s'il y a lieu de les mettre en rapport avec une réalité différente ou «ultime».

Pritchard soulignera encore, entre autres, une certaine inauthenticité du sceptique radical, qui (comme le relativiste en matière de vérité) ne conforme pas sa vie à ses prétendues convictions; ce qui apparaît dès lors comme une posture, loin de menacer comme on le dit parfois les structures de pouvoir existantes, ne fait que les affermir. L'auteur d'un livre aussi bref ne peut développer ni même aborder tous les aspects du thème qu'il étudie. Certes, on pourra s'étonner qu'un ouvrage introductif sur le scepticisme (en langue anglaise, qui plus est) ne cite pas une seule fois le nom de Hume, mais le parti pris de Pritchard fait la force de son propos: il n'a pas voulu survoler son sujet mais éclairer la question — l'énigme — qui lui a paru la plus intéressante, la plus aiguë. Il y a parfaitement réussi. 